\title{
Using a Cell-based WLAN Infrastructure Design for Resource-effective and Accurate Positioning
}

\author{
Eddie C.L. Chan, George Baciu, S.C. Mak
}

\begin{abstract}
A large scale WLAN infrastructure requires the placement of many thousands of access points (APs). The current approach is to deploy these in an empirical and ad-hoc manner. However, this deployment results in poor resource utilization and inaccurate positioning due to signal overlap and black spots. In this paper, we propose three structured approaches to WLAN infrastructure deployment that would allow better positioning accuracy and optimal coverage. These three approaches make use of triangular, square, and hexagonal configurations. Our results show that all three are more effective in both 2-D and 3-D space than any of the current ad-hoc or empirical approaches to AP deployment. Overall, the hexagonal approach is the most cost effective and accurate. It allows better positioning with fewer APs than are normally used. As a further contribution, 3-D rendering of buildings and wireless signal coverage could give engineers a concrete visualization that helps them to foresee where the blind spots are in advance and how signal varied across multi-story buildings, such that engineers could estimate the optimal number of APs and where they should be placed.
\end{abstract}

Index terms: WLAN infrastructure, location fingerprinting, localization

\section{INTRODUCTION}

Wireless Local Area Networks (WLANs) are currently deployed on a large-scale and in a wide range of urban environments. Very often, these WLANs are made up of many access points (APs) or nodes deployed across extensive, topographically varied, heavily built up, and constantly changing environments that may carry heavy traffic [1]. The basic requirements of effective WLANs are that they should provide adequate coverage so that when users wish to access location-aware (e.g., pervasive computing-enabled) applications and services, the WLANs will permit user mobile devices to be accurately positioned and that WLANs be deployed in a cost-effective and resource-efficient way, both in outdoor and indoor environments. Current approaches to infrastructure design and deployment, however, apply an unstructured approach to WLAN infrastructure design that implies poor resource utilization, placing and positioning APs manually on the basis of empirical measurements of RSS (received signal strength) taken by engineers. [2]. Such an approach may use many APs but still leave blind spots or places where there are too many access points packed closely together. An ad-hoc AP deployment results in signal overlap and interference reducing positioning accuracy.

Manuscript received Aug. 23, 2009; revised November 10, 2009.

Authors are with the Department of Computing, The Hong Kong Polytechnic University Hung Hom, Kowloon, Hong Kong Email: \{csclchan, csgeorge,csscmak\}@comp.polyu.edu.hk
Current research on the visual representation of WLAN signals $[3,4]$ is based on the accuracy of positioning systems and proximity graphs, such as the Voronoi diagram and clustering graphs. There are two drawbacks to these representations. First, none of them allow the visualization of location uncertainty. For example, showing on a 2-D or 3-D display where there might be blind spots would be paramount to the initial deployment of APs. Second, these methods are difficult for the estimation of the AP number required for optimal coverage. Also, the dynamic location optimization of APs is still an open problem.

Location or positioning uncertainty could be identified where signals overlap. In our simulations we use a location fingerprinting (LF) [4-8] approach. In that case, positioning uncertainty occurs within the locus, a line (a series of points) having the same RSS measured within the overlap of two or more AP signals. This identity of RSS makes it impossible to accurately localize points of uncertainty. Increasing the number of APs in an area reduces the zone of most common overlap and thereby shortens the locus (seen as a line). Given enough APs, positioning uncertainty can be eliminated and it becomes possible (in terms of a particular resolution) to achieve perfect positioning. But as long as an ad-hoc approach is used, it is not possible to estimate in advance the optimal number of APs. A more structured approach should produce economies and efficiencies as well as improved capabilities. In our previous work $[9,10]$, we focused on the wireless tracking problem from the view of the end-user. We enhanced the positioning algorithm of the traditional location fingerprinting approach with fewer access points and make more effective to achieve accurate positioning.

In this paper, we focus on how to achieve accurate positioning from the view of wireless infrastructure deployment. We propose a cell-based WLAN infrastructure deployment design that allows the visualization of blind spots, predicts how many APs will be optimally required for positioning, and suggests where they should be placed. The proposed approach extends our previous work $[11,12]$ from a two-dimensional to a three-dimensional approach and uses three structured geometric configurations for use in WLAN infrastructure deployment: (1) triangular, (2) square, and (3) hexagonal.

The proposed approach both improves the speed and efficiency of large-scale WLAN infrastructure deployment and allows WLAN engineers and designers to choose an optimal deployment solution from a selection of different AP distributions. Our simulations show that all of the proposed structured approaches are much more cost effective than the current unstructured AP deployment approach, all producing a regular and predictable location tracking. The hexagonal 
approach is overall the most cost-effective way to achieve perfect accuracy, requiring only two-thirds as of the APs required by the unstructured approach. As a further contribution, we offer a virtual, 3-D rendering of wireless signal coverage in both a small-scale laboratory environment and a large-scale university campus environment. It would be useful in any kind of built-up area but in particular where there are many multi-story buildings.

The rest of this paper is organized as follows: Section II describes related work on the deployment of mobile phone networks and contrasts it with our approach. Section III defines location uncertainty. Section IV presents the design of the proposed cell-based WLAN infrastructure. Section V describes the positioning methodology. Section VI introduces channel interference among APs. Section VII presents the visualization of WLAN signal distribution. Section VIII presents the simulation setup. Section IX presents the results and discussion. Finally, Section X offers our conclusion and future work.

\section{RELATED WORK}

Although mobile phone networks do not require highly accurate positioning, they do address a very similar set of problems to those of WLAN AP positioning in that they must efficiently and cost-effectively cover an area with a signal of adequate strength. The current mobile network approach is to establish a regularly-placed infrastructure of mobile stations (MS) [13]. Each MS is placed at the centre of a hexagon that lies within the radius of the signal of the MS and that is contiguous with other similar hexagons so as to form a honeycomb-celled tessellation over the network area.

While the requirements and constraints of the mobile cell network and an AP network are similar, they are not identical in at least five ways. First, black spots and interference are more tolerable in cell phone networks because mobile users can very quickly move into areas of more acceptable signal strength or quality. Second, MS apparatus are much more powerful than AP devices and so are less limited in their ability to pass through walls or are less influenced by traffic or by other signaling devices. Third, MS devices may be licensed to override competing signals. Fourth, a very large mobile area can be covered by a relatively small number of MS, while a WLAN (Wi-Fi) network will require a proportionally much larger number of APs. Finally, and perhaps most distinctively, positioning is qualitatively different in the two technologies. In a mobile network, devices can be located merely as being within the radius of an MS. In a Wi-Fi network, we can locate a device much more accurately, as being at a particular coordinate. Currently, this requires that the device be in communication with two or more APs so as to be able to calculate data from them. However, in certain real-world scenarios, a device will not be simultaneously within the range of more than two APs. Consequently, while it might be able to recognize a device in its radius, it may not be able to calculate its coordinates.

\section{PRoBlem Definition OF LOCATION UNCERTAINTY}

Location uncertainty could be identified when one of the coordinates in a 2-D or 3-D space could not be given by the sensors. In this paper, we make use of existing WLAN infrastructure to estimate the position of a WLAN-enabled device. There are two fundamental positioning approaches, propagation-based $[6,14,15]$ and location fingerprinting (LF) [5-7]. We will use an LF approach only in following examples.

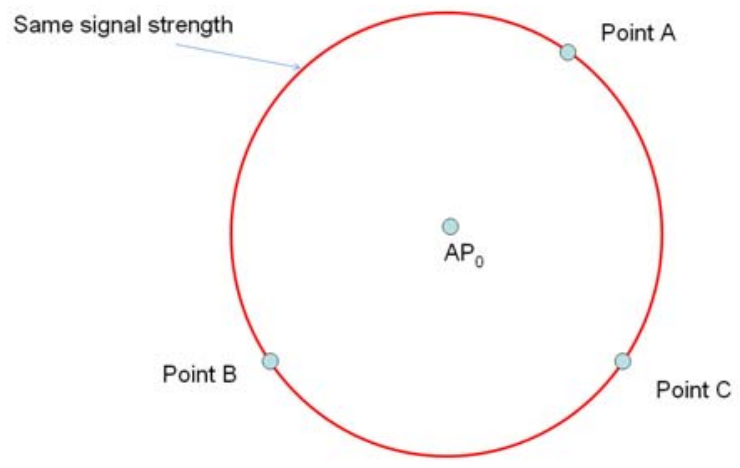

Fig. 1. Location uncertainty with one access point case is on the circumference of signal propagation where they share same RSS.

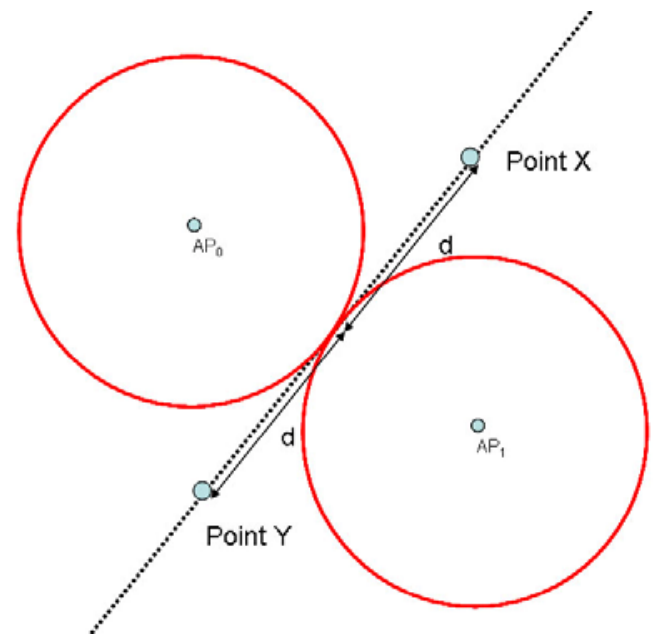

Fig. 2. Location uncertainty with 2 access points case is on the tangent of circular signal propagation where they share same RSS.

LF-based approaches [4-7] locate a device by accessing a database containing the location fingerprint (RSS and coordinates) of other devices within the Wi-Fi footprint and then uses those fingerprints to calculate its own coordinates compared with those in an LF database. More specifically, the LF method requires the collection of data $\left\{\left(Y_{n}, C_{i}\right), i=1 \ldots N\right\}$, for $\mathrm{N}$ locations in the site, where $\mathrm{C}_{\mathrm{i}}$ is the known location of the $i$ 'th measurement and $\mathrm{Y}_{\mathrm{i}}=\left(\mathrm{Y}_{\mathrm{i}}, \ldots, \mathrm{Y}_{\mathrm{iN}}\right)$ is the RSS vector when the transmitter is at $\mathrm{C}_{i}$. The vector $\mathrm{Y}_{\mathrm{i}}$ is the "fingerprint" of the location $C_{i}$. When a new fingerprint $Y$ is observed from a transmitter in unknown location $\mathrm{A}$, the locations of $\mathrm{Y}$ is estimated by searching for the fingerprint $\mathrm{Yi}$ that is closest to $\mathrm{Y}$ at $\mathrm{d}$ distance and estimate the location with the corresponding $\mathrm{C}_{\mathrm{i}}$.

When using an LF approach in a WLAN infrastructure, location uncertainty can be defined as a locus - a line (a series of points) having the same RSS measured within the overlap of two or more AP signals. Fig. 1 shows the problem with one AP and circular signal propagation. Points $\mathrm{A}, \mathrm{B}$ and $\mathrm{C}$ share the same RSS (location fingerprint). If a user is located at point B, the LF approach would not be able to estimate whether the user is at point $\mathrm{A}, \mathrm{B}$ or $\mathrm{C}$. In the case of one $\mathrm{AP}$, the location uncertainty happens on the circumference of signal 
propagation. Fig. 2 shows the problem where two APs, at points $\mathrm{X}$ and $\mathrm{Y}$, share the same location fingerprint at a point on a tangent and so share the same RSS and cannot be located.

As noted earlier, a solution is to increase the number of APs in the area, reducing the zone of most common overlap, and shortening the locus. Given enough APs, location uncertainty can be eliminated. But such an ad-hoc approach would not be able to tell us in advance how many APs are optimally required or where they should be placed.

Uncertainty is usually derived from the geometric dilution of precision in GPS [16, 17]. Dilution of precision provides a scaling factor for the GPS receiver's accuracy. [16, 17] quantify the location uncertainty in meters. In our definition, location uncertainty is a co-ordinate in a 2-D or 3-D space that could not be identified its location. In Section IX, we base on WLAN signal coverage and calculate every co-ordinate of location uncertainty.

\section{THE DESIGN OF THE CELL-BASED WLAN INFRASTRUCTURE}

A maximally accurate and resource-efficient cellular WLAN infrastructure will cover the biggest area with the minimum number of APs without allowing empty spaces and with the minimum of interference between cells. A tessellation is thus required and this can be achieved using regular polygons of three kinds: quadrangles, triangles, and hexagons. Each AP is placed at the center of the cell. A hexagonal plane tessellation will produce a honeycomb mesh. [18] Fig. 3, Fig. 5 and Fig. 7 show a 2-D distribution of APs in triangular, square, and hexagonal tessellations. Fig. 4, Fig. 6 and Fig. 8 show triangular, square, and hexagonal distributions of APs in 3-D space. The red points indicate the AP locations, the green regions are the cell area, and the yellow spheres represent the wireless coverage of the APs.

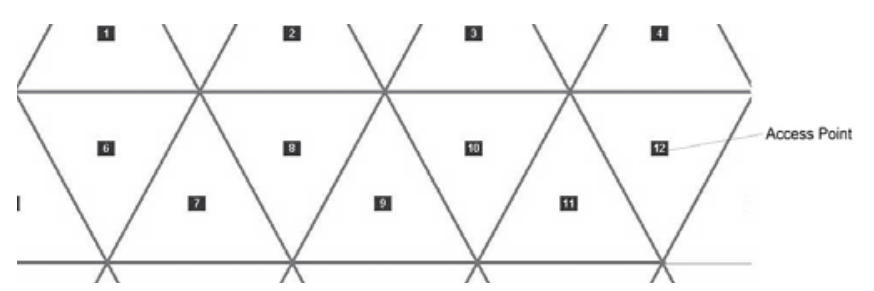

Fig. 3. Triangular Tessellation

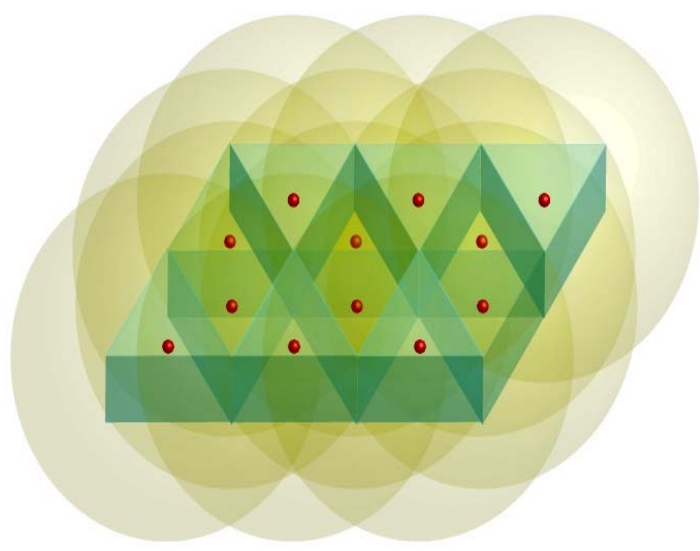

Fig. 4. Triangular distribution

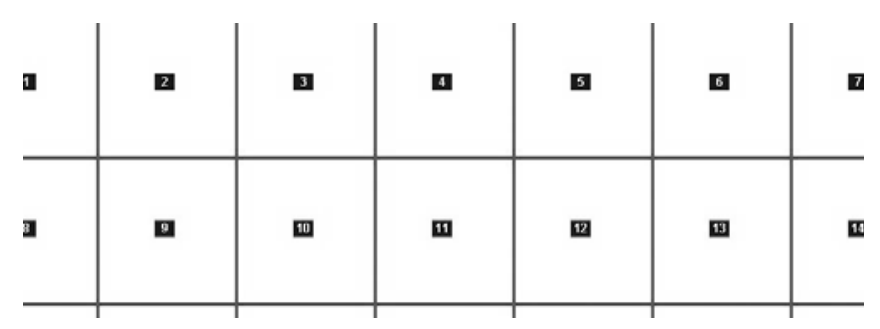

Fig. 5. Square Tessellation

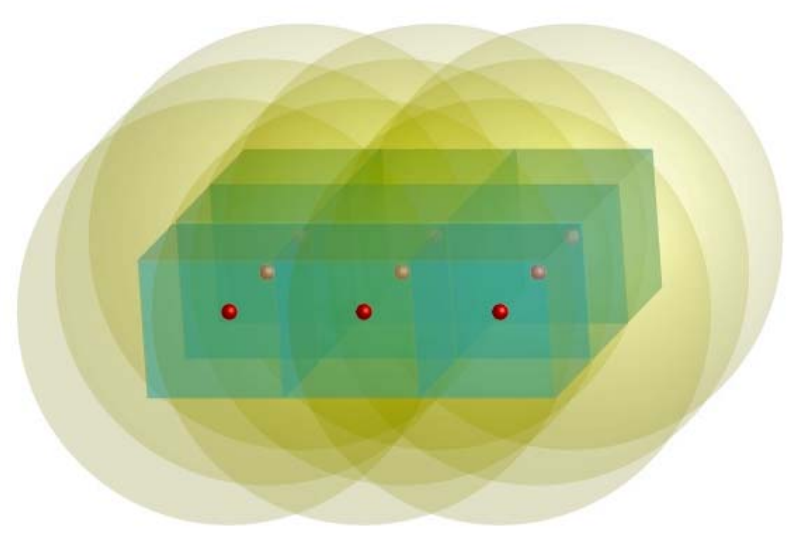

Fig. 6. Square distribution

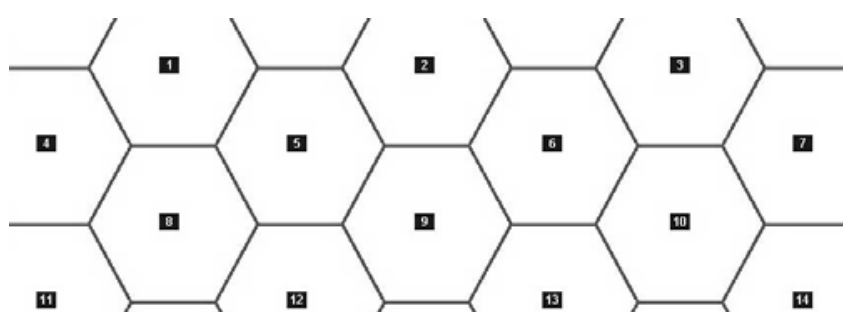

Fig. 7. Hexagonal (or honeycomb mesh) Tessellation

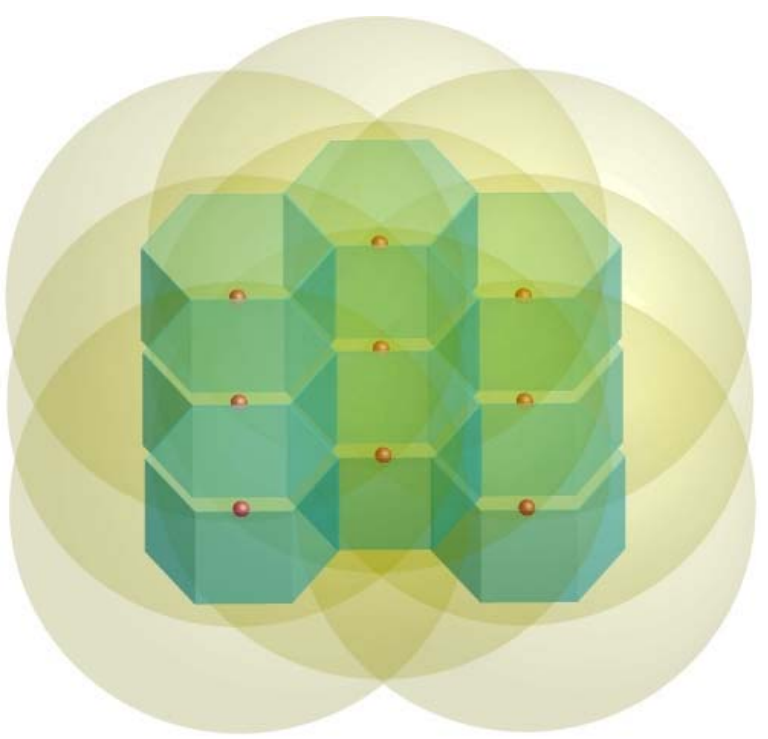

Fig. 8. Hexagonal distribution

Consider a physical layout of the wireless network as shown in Fig. 7. The hexagonal area is a cell. Each cell will contain 
one AP and for a simplistic reason, we consider to have a circular Wi-Fi coverage. Thus, there are signal overlap areas. It may not be possible to localize a device in such an area dues to the locus problem as described in the Introduction and Section III. In an LF-based approach, they deal with the positioning uncertainty problem by estimating the location using a prerecorded LF database for each location. The positioning accuracy can be defined as the number of distinguishable location points. On this basis, then, APs would be evenly distributed, placed at the centre of hexagons as in Fig. 9. In this configuration, each AP provides total coverage of seven cells, i.e., its own and six contiguous cells, and a user receives at least seven AP signals.

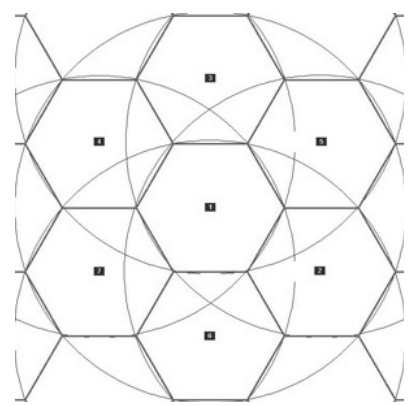

Fig. 9. Hexagonal Cells satisfy with 1.5 cell radius coverage

\section{The Positioning Methologies}

Two positioning methodologies are typically applied in WLANs, propagation based approaches and location fingerprinting (LF). Our previous works $[9,10]$ make use of LF to track a WLAN-enabled device. In our later simulations we will use only an LF approach but for completeness in the following we briefly describe both.

\section{A. Propagation-based Approach}

Propagation based approaches $[14,15]$ estimate the position of a device by first applying an algorithm to calculate the RSS of APs in an area and then applying a set of triangulation algorithms. The propagation-based algorithm [7] is used to calculate the RSS as follows:

$$
r_{i}\left(d_{k}\right)=r_{0}\left(d_{0}\right)-10 \alpha \log _{10}\left(d_{k}\right)-\text { wallLoss }
$$

where $D=\left\{d_{1} \ldots d_{n} \mid d_{i} \in \mathfrak{R}^{3}\right\}$ is a set of locations; $R=\left\{r_{1} \ldots r_{n} \mid r_{i} \in \mathfrak{R}^{n}\right\}$ is a set of current received LF vectors with respect to known $d_{i}$; $\alpha$ is the path loss exponent (clutter density factor) and wallLoss is the sum of the losses introduced by each wall on the line segment drawn at Euclidean distance $d_{k}$.

Initially, $r_{0}$ is the initial RSS at the reference distance of $d_{0}$ is 1 meter (this is $41.5 \mathrm{dBm}$ for LOS propagation and for 37.3 $\mathrm{dBm}$ NLOS propagation for some report measurement) [19]. The path loss exponent $\alpha$ at a carrier frequency of $2.4 \mathrm{GHz}$ is reported to be 2 for LOS propagation and 3.3 for NLOS propagation [7]. Under other circumstances, $\alpha$ can be between 1 and 6.

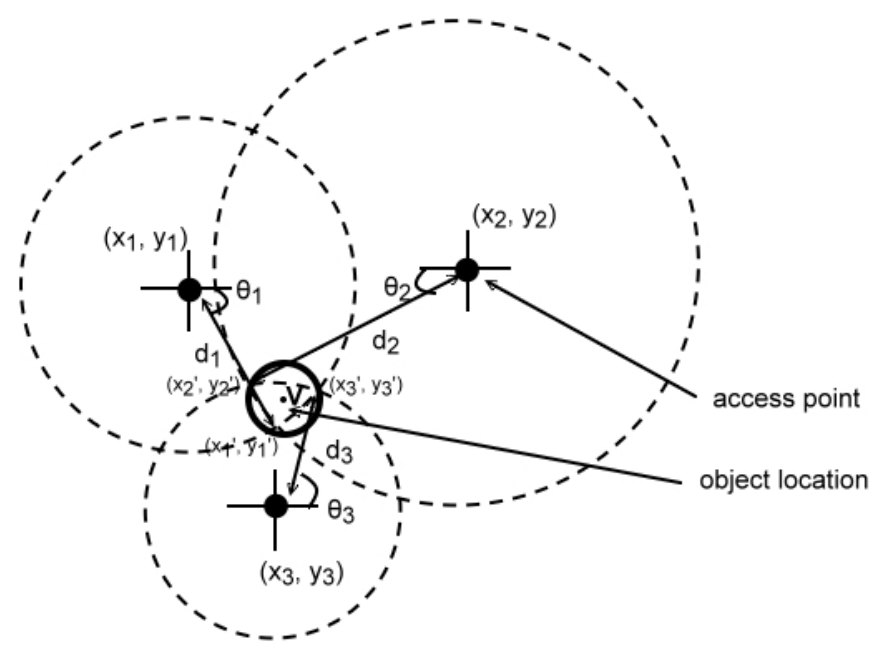

Fig. 10. Triangular Algorithm

The triangular positioning algorithm uses trigonometry and geometry to compute the locations of objects. In a 2D environment, this requires three access points (APs). The locations of these three APs' location are denoted as $\left(x_{1}, y_{1}\right)$, $\left(x_{2}, y_{2}\right),\left(x_{3}, y_{3}\right)$ and the object location is $(x, y)$. Using the propagation-based theorem in (1), we can denote the distance between the access points and object location as $d_{1}, d_{2}$, and $d_{3}$, where $d_{0}$ is the initial RSS at the reference distance. To estimate the location of the object, we use the tri-lateration method as follow.

$$
\left\{\begin{array}{l}
d_{1}=d_{0} 10^{\frac{r_{0}-r_{1}-\text { wallLoss }}{10 \cdot \alpha}} \\
d_{2}=d_{0} 10^{\frac{r_{0}-r_{2}-\text { wallLoss }}{10 \cdot \alpha}} \\
d_{3}=d_{0} 10^{\frac{r_{0}-r_{3}-\text { wallLoss }}{10 \cdot \alpha}}
\end{array}\right.
$$

After calculating the distance, we find the angle $\theta_{1}, \theta_{2}$ and $\theta_{3}$ between the object location and APs, and then we are able to calculate the possible location matrix of the object as follows:

$$
\left(\begin{array}{c:c}
x_{1}{ }^{\prime} & y_{1}{ }^{\prime} \\
x_{2}{ }^{\prime} & y_{2}{ }^{\prime} \\
x_{3}{ }^{\prime} & y_{3}{ }^{\prime}
\end{array}\right)=\left(\begin{array}{c:c}
x_{1}+d_{1} \cos \theta_{1} & y_{1}+d_{1} \sin \theta_{1} \\
x_{2}+d_{2} \cos \theta_{2} & y_{2}+d_{2} \sin \theta_{2} \\
x_{3}+d_{3} \cos \theta_{3} & y_{3}+d_{3} \sin \theta_{3}
\end{array}\right)
$$

If three circles does not intersect at a point, each column value of a location matrix are different, we could simply average the matrix value to estimate the location $(x, y)$ as follows:

$$
x=\frac{x_{1}{ }^{\prime}+x_{2}{ }^{\prime}+x_{3}{ }^{\prime}}{3}
$$




$$
y=\frac{y_{1}{ }^{\prime}+y_{2}{ }^{\prime}+y_{3}{ }^{\prime}}{3}
$$

\section{B. Location Fingerprinting Approach}

There are two alternative Location Fingerprinting approaches, the K-Nearest Neighbor (K-NN) and the probabilistic approach.

\section{1) K-Nearest Neighbor Location Fingerprinting Approach}

The K-Nearest Neighbor (K-NN) algorithm requires two sets of data. The first set of data is the samples of RSS from $N$ APs in the area. Each element in a vector is an independent RSS (in $\mathrm{dBm})$ collected from APs in the location. The second set of data contains all of the average RSS from $N$ APs at a particular location. This second dataset forms the location fingerprinting database. $F=\left\{f_{1} \ldots f_{n} \mid f_{i} \in \mathfrak{R}^{n}\right\}$ is a set of sampling LF vectors in database. We estimate the location $d_{k}$ by clustering the Euclidean distance $\left|r-f_{i}\right|$ between current received LF vector $r$ and sampling LF vector $f_{i}$ with position $d_{i}$ as

$$
d=\frac{\sum_{i=0}^{n} \frac{d_{i}}{\left|r-f_{i}\right|}}{\sum_{i=0}^{n} \frac{1}{\left|r-f_{i}\right|}}
$$

\section{2) Probabilistic Location Fingerprinting}

Probabilistic LF applies Baye's theorem to calculate the most probable location out of the pre-recorded LF database, $F=\left[f_{l}\right.$, $\left.f_{2}, f_{3} \ldots f_{N}\right]$. We can estimate $d$ by

$$
\arg \max _{d}[P(d / F)]=\arg \max _{d}\left[\frac{P(F / d) P(d)}{P(F)}\right]
$$

Since $P(F)$ is constant for all $d$, the algorithm can be rewritten as

$$
\arg \max _{d}[P(d / F)]=\arg \max _{d}[P(F / d) P(d)]
$$

As $P(d)$ can be factored out from the maximization process, the probabilistic positioning algorithm is as

$$
P(F / d)=\prod_{i=1}^{N} P\left(f_{i} / d\right)
$$

\section{OVERLAPPING CHANNEL INTERFERENCE}

The bandwidth of wireless network is limited because of the property of wireless networks and stations have to share the limited bandwidth. [20] IEEE $802.11 \mathrm{~b} / \mathrm{g}$ has 14 overlapping frequency channels. Channel 1, 6 and 11 are non-overlapping channels.

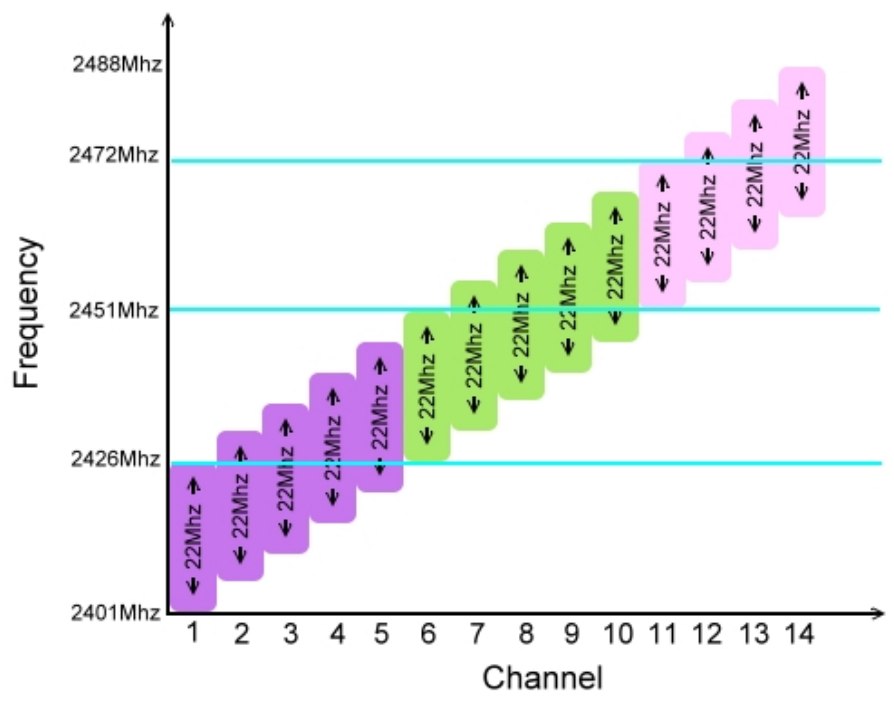

Fig. 11. IEEE $802.11 \mathrm{~b} / \mathrm{g}$ Frequency Spectrum to Channel Number

As shown in Fig. 11, IEEE 802.11 b/g spreads through 2,401 $\mathrm{MHz}$ to 2,483 MHz. Each channel spreads over $22 \mathrm{MHz}$. Only $5 \mathrm{MHz}$ separates two adjacent channels such that most of existing channels are overlapped.

\section{A. Interference-level Factor}

The interference-level factor $\gamma$ is defined as follows:

$$
\gamma(\Delta c)=\max (0,1-k \Delta c)
$$

where $\Delta c$ is the absolute channel difference and $k$ is the nonoverlapping ratio of two channels. $\gamma$ and $\Delta c$ are in $\mathrm{Db}$ unit. When $\Delta c$ increases, $\gamma$ decreases. For example, if $\Delta c=0$, then $\gamma(\Delta c)=1$ and if $\Delta c \geq 5$, then $\gamma(\Delta c)=0$. In other words, for channel 1 and $6, \Delta c=5, k=0$, then $\gamma(\Delta c)=0$, suggesting no interference. In real case, if APs are installed far enough with others, $\gamma$ should be at least equal to the above threshold.

\section{B. Signal-to-Interference-plus-Noise-Ratio}

Signal-to-Interference-plus-Noise-Ratio (SINR) is a very common indicator to measure interference. SINR is defined as follows:

$$
S I N R=\frac{R_{b}}{\gamma(\Delta c) \sum R+n}
$$

where $R_{b}$ is the highest RSS after path loss calculation. $R$ is the remaining set of RSS after path loss calculation. $n$ is the noise signal strength. $R_{b}, R, n$ are in $\mathrm{dBm}$ unit. Usually, $n$ should have the value of $-100 \mathrm{dbm}$. Again SINR should be at least equal to above calculated threshold which depends on the distance among APs, the transmission rate, the modulation scheme and the required bit-error rate. 


\section{WLAN SIGNAL DISTRIBUTION}

In this section we extend our previous work [8] and describe our approach to visualizing the distribution of a WLAN signal.

We first conducted a survey of WLAN signals on the campus of The Hong Kong Polytechnic University (PolyU). The approximate area of the campus is 9.34 hectare and there are 26 main buildings from Core $A$ to Core $Z$ and seven other buildings with WLAN access. The sampling schedule was set to collect the RSS data every 5 seconds. The vector of the RSS data at each location forms the location fingerprint with around 20 RSS elements in the vector. We measured the signal at 27 locations on the campus. The total number of RSS samples was 12 days $\mathrm{X} 4$ directions $\mathrm{X} 27$ buildings $\mathrm{X} 20$ APs X 2 times $=$ 51840. Fig. 12 shows a 3-D rendering of parts of the PolyU campus.

On the top of the 3-D PolyU campus model, we superimposed the WLAN signal distribution color-coded as follows: red represents strong signals and blue weak signals. 3$\mathrm{D}$ rendering of buildings and wireless signal coverage could give engineers a very concrete visualization that helps them to estimate the signal distribution in advance and plan for deployment of wireless infrastructure.

The following normalization distribution is used to represent the fuzzy membership functions.

$$
P(x)=\frac{1}{\sqrt{2 \pi} \sigma} e^{\frac{(x-\mu)^{2}}{2 \sigma^{2}}}
$$

where $\mathrm{p}(\mathrm{x})$ is the probability function, $\mathrm{x}$ is the normalized RSS, $\sigma$ is the standard deviation of normalized signal normalized strength in a region, $\mu$ is the mean of signal strength in a region. The wireless network covers the entire campus. The fuzzy set interval of blue is $[0,0.5],[0,1]$ is green and $[0.5,1]$ is red. For the blue region, we substitute $\sigma=0.5, \mu=0$.

$$
\mu_{\text {Blue }}(0<x<0.5)=\frac{2}{\sqrt{2 \pi}} e^{-2 x^{2}}
$$

For the green region, we substitute $\sigma=0.5, \mu=0.5$.

$$
\mu_{\text {Green }}(0<x<1)=\frac{2}{\sqrt{2 \pi}} e^{-2\left(x-\frac{1}{2}\right)^{2}}
$$

For the red region, we substitute $\sigma=0.5, \mu=1$.

$$
\mu_{\operatorname{Re} d}(0.5<x<1)=\frac{2}{\sqrt{2 \pi}} e^{-2(x-1)^{2}}
$$

Assume that $\mathrm{B}$ is a finite set of RSS vector belonging to a particular color region, where $B=\left\{b_{1} \ldots b_{n} \mid b_{i} \in \mathfrak{R}^{n}\right\}$, i.e., $b_{i} \in S, \forall S \in R$, and $\forall S \in[l, u]$, where $l$ is the lower bound of fuzzy interval and $u$ is a upper bound of fuzzy interval. To analyze the distribution surfaces $\mathrm{S}$, there always exists a spatio-temporal mapping, $q: B \rightarrow S$.

$$
q(x)=\int_{S} h(x) b(S) d S
$$

where $h(x)$ is the characteristic function of $\mathrm{S}$, i.e.,
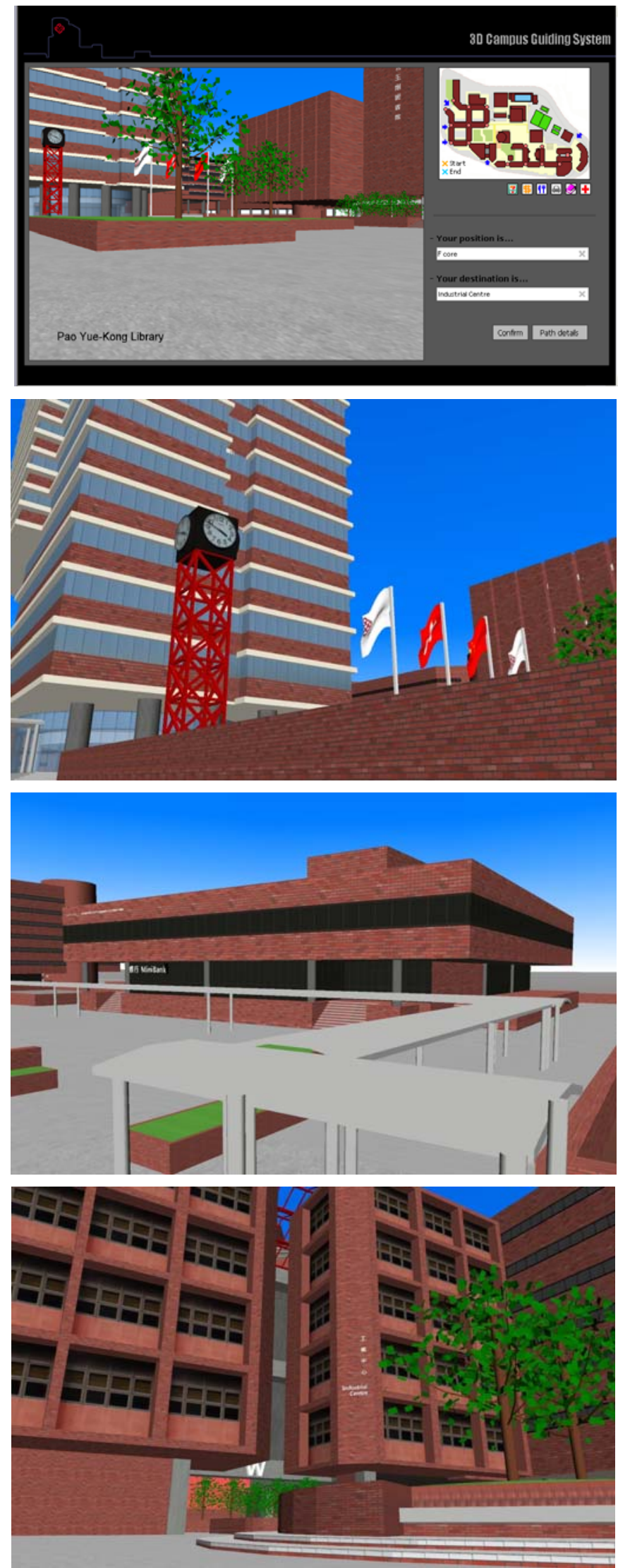

Fig. 12. 3-D PolyU Campus 


$$
h(x)= \begin{cases}1 & x \in S, \\ 0 & x \notin S\end{cases}
$$

and $b(S)$ is a weight function that specifies a prior on the distribution of surfaces $S$. We can explicitly define $b(S)$ by (1). By (13), (14), (15), (16) and (17), the WLAN signal distribution can be illustrated. Fig. 13 shows the 3-D Fuzzy WLAN signal Distribution. The signal can be received around $75 \mathrm{~m}$ height and cover almost every place of PolyU campus.

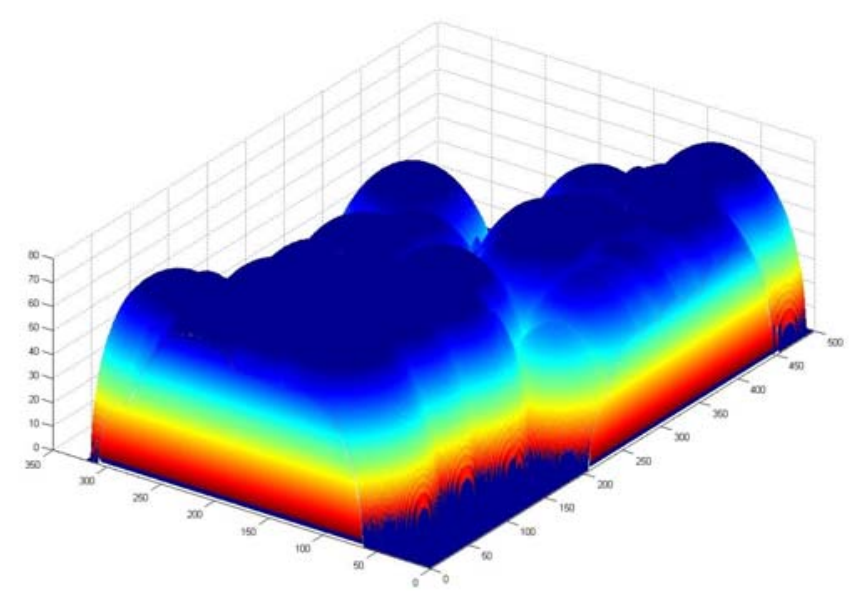

Fig. 13. 3-D Fuzzy WLAN Signal Distribution with the campus floor plan

\section{SIMULATION SETUP}

In the following sections we describe two sets of simulations. The first set of simulations (Set A) is designed to determine the positioning accuracy of our four basic distributions, triangular, square, hexagonal and ad-hoc where we vary the number of APs and interference. Furthermore, we investigate 2-D and 3-D distribution of location uncertainty under different structured approaches. The second of simulations (Set B) is designed to have the proposed $\mathrm{Wi}-\mathrm{Fi}$ tracking system on the 7th floor of the PQ building, in Department of Computing, at The Hong Kong Polytechnic University.

In all two sets of simulations, for reasons that have been mentioned, we measured accuracy using only the LF approach Accuracy is defined in a range from zero to one, with zero meaning not detected and one meaning perfectly accurate. The radius of coverage of each AP is $80 \mathrm{~m}$. In Set $A$, the signal strength ranges between $-85 \mathrm{dbm}$ and $-30 \mathrm{dbm}$ in a testing space of $150 \mathrm{~m}$ X $100 \mathrm{~m} \times 10 \mathrm{~m}$. Table I summarizes the setup for Set A.

In Set B, we walked through the hallway on the $7^{\text {th }}$ Floor with a WLAN-enabled device. The estimated and actual coordinates were calculated and collected at 61 locations in the hallway. The dimension of floor is approximately $50 \mathrm{~m}$ by $20 \mathrm{~m}$. The received signal sensitivity also limits the range of the RSS to be between $-90 \mathrm{dBm}$ and $-35 \mathrm{dBm}$. Table II summarizes the setup for Set B.
TABLE I.

Simulation Settings (SET A)

\begin{tabular}{|c|c|}
\hline Item & Description \\
\hline Total area & $150 \mathrm{~m} \times 100 \mathrm{~m}$ \\
\hline Height & $10 \mathrm{~m}$ \\
\hline Positioning resolution & 3 meters \\
\hline Wi-Fi coverage from each APs & 80 meters \\
\hline Range of signal strength & $-85 \mathrm{dbm}$ to $-30 \mathrm{dbm}$ \\
\hline
\end{tabular}

TABLE II.

SUMMARY OF EXPERIMENT (SET B)

\begin{tabular}{ll}
\hline Item & Description \\
\hline \hline Total laboratory area & 1000 square meter \\
\hline Number of sample points & 4880 sample points \\
\hline Positioning resolution & 3 meters \\
\hline Wi-Fi channel & 1,6, and 11 \\
\hline Positioning resolution & 3 meters \\
\hline Range of signal strength & $-90 \mathrm{dBm}$ to $-35 \mathrm{dBm}$ \\
\hline
\end{tabular}

\section{IX.RESULTS AND DISCUSSION}

In this section we describe the result from the simulation Sets A and B. Subsection A to D report the result in Set A. Subsection E reports the result of trajectory estimation in Set B. Subsection $F$ reports the signal distribution in a real-case environment.

\section{A. Effect of cell-based WLAN infrastructure and number of APs to Accuracy}

Fig. 14 and Table III show the relationship of number of APs to positioning accuracy under the four different deployment scenarios. As can be seen in Fig. 14, the performance of the four approaches is similar when few APs are used yet the structured approaches all nonetheless always outperform unstructured approaches at every setting. The superior performance of the structured approaches becomes progressively stronger as more APs are added and ultimately they achieve perfect accuracy. Except at very low numbers of APs, when the triangular approach is the most accurate, the hexagonal distribution is the most accurate approach and achieves perfect accuracy first, at 18 APs.

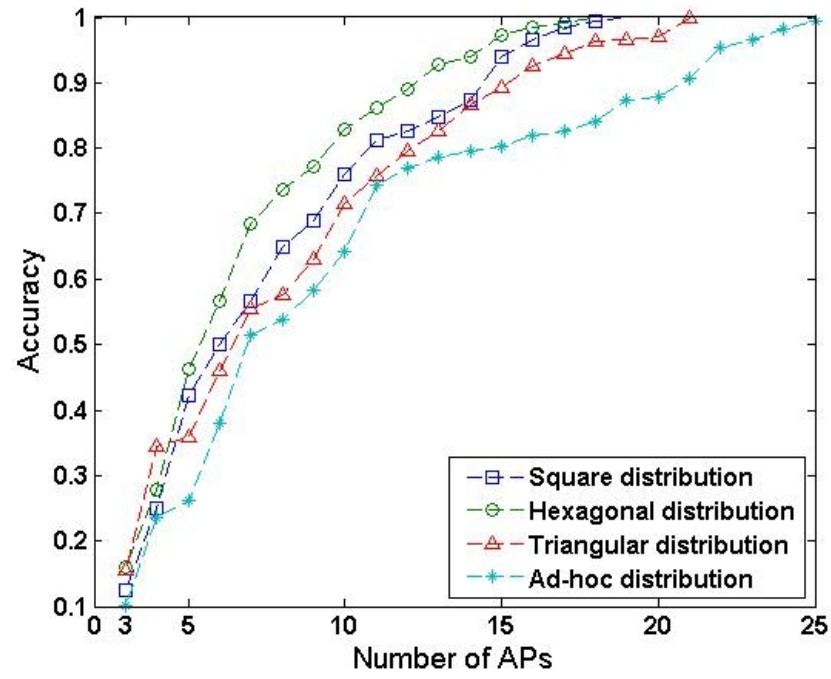

Fig. 14. Relationship of number of APs to positioning accuracy under four differnt deployment scenarios 
Table III compares the cost-efficiency of the structured and the ad-hoc or empirical approaches. The hexagonal approach is clearly the best performer, achieving perfect accuracy with just 18 APs, making it considerably more cost-effective than the next-most effective, square, and very much more effective than triangular, which requires more than $40 \%$ more APs to achieve the same accuracy. Perhaps the most important point to take away here is that the unstructured approach never at any point matches the accuracy of the structured approaches and never achieves perfect accuracy. In fact, the best performance of the unstructured approaches, just 96\%, was achieved over 100 iterations and still required $23 \mathrm{APs}$, the number of APs required by the worst of the structured performances.

TABLE III.

Positioning Accuracy When VARying Number of APs

\begin{tabular}{lllll}
\hline \multirow{2}{*}{ No. of APs } & \multicolumn{4}{l}{ Accuracy (\%) } \\
\cline { 2 - 5 } & Triangle & Square & Hexagon & Ad-hoc \\
\hline \hline 2 & $6 \%$ & $7 \%$ & $8 \%$ & $4 \%$ \\
\hline 5 & $36 \%$ & $42 \%$ & $46 \%$ & $26 \%$ \\
\hline 10 & $72 \%$ & $76 \%$ & $82 \%$ & $64 \%$ \\
\hline 15 & $89 \%$ & $93 \%$ & $97 \%$ & $80 \%$ \\
\hline 16 & $92 \%$ & $96 \%$ & $98 \%$ & $82 \%$ \\
\hline 18 & $96 \%$ & $99 \%$ & $100 \%$ & $84 \%$ \\
\hline 20 & $97 \%$ & $100 \%$ & $100 \%$ & $88 \%$ \\
\hline 23 & $100 \%$ & $100 \%$ & $100 \%$ & $96 \%$ \\
\hline$>25$ & $100 \%$ & $100 \%$ & $100 \%$ & $99 \%$ \\
\hline
\end{tabular}

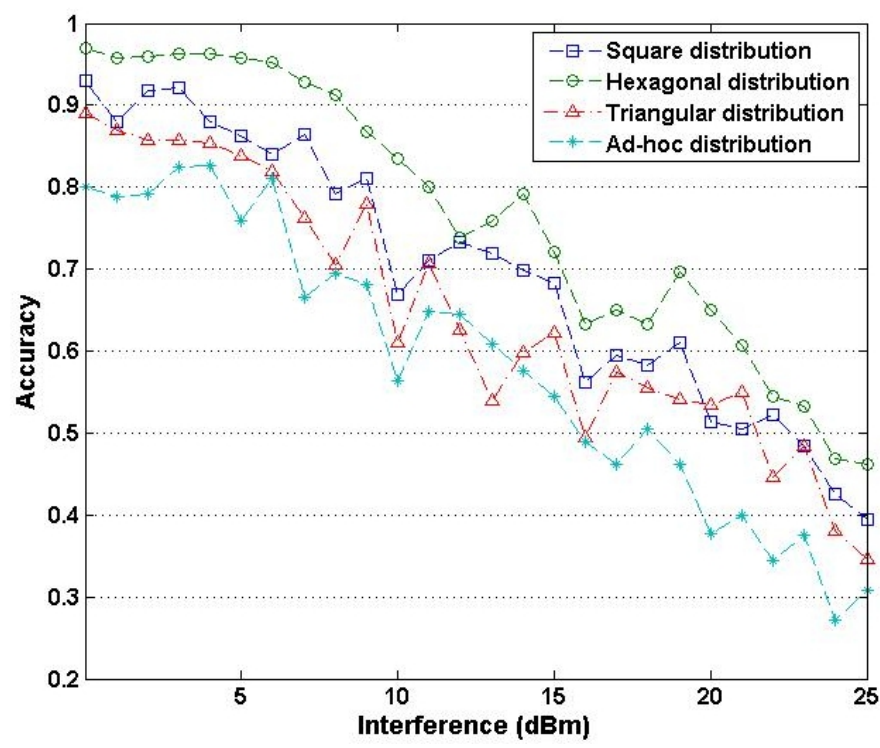

Fig. 15. Relationship of interference to accuracy under ascending, orthogonal and ad-hoc channel allocation

\section{B. Effect of Interference on the positioning accuracy}

Fig. 15 shows the relationship for the channel interference to positioning accuracy. In order to see how channel interference affected the positioning accuracy specifically, we fix the number of APs to be 13 . We only vary the channel interference from 0 to $25 \mathrm{dBm}$ and the accuracy is in a scale from 0 to 1 (1 represents $100 \%$ accuracy). The channel interference obeys (10) and (11). Four different types of access point distribution have over $80 \%$ positioning accuracy when the interference is below $7 \mathrm{dBm}$. When the interference strength increases after $10 \mathrm{dBm}$, the performance of positioning accuracy worsens seriously.
Obviously, when channel interference increases, the positioning accuracy decreases. However, this interference value is difficult to control because it depends on the environment. One way of improving this is to take more iteration. This could contribute to adjust the setting. As can be seen in Fig. 15, the positioning performance of hexagonal distribution is the most accurate. The result indicates that hexagonal distribution could effectively achieve approximately $12 \%$ more accurate when the system is under high channel interference.

\section{Result of 2-D Simulation of Location Uncertainty under triangular,square and hexagonal distribution}

The colored dots represent positions of the localization uncertainty. Each pair of colored dots represent where localization returned same RSS reading for two coordinates. In other words, more colored dots means the positioning accuracy is worse. Each blue arc line represents the coverage of an AP.

Fig. 16, Fig. 17, and Fig. 18 represent WLAN infrastructure AP deployments under the simulation setup for our three proposed geometric deployment patterns. The testing area is $3 \times 3 \mathrm{~m}$ 2-D grid. There are 1,734 grid points. The colored dots in corners show places where accurate positioning is not possible. Fig. 16 (triangular) shows 83 colored points and the overall positioning accuracy is $95.2 \%$. Fig. 17 (square) shows 52 colored points and a positioning accuracy of 97\%. Fig. 18 (hexagonal) shows 39 colored points and a positioning accuracy of $97.8 \%$. The performances are similar to what was found in SET A. A hexagonal distribution of APs gets the highest accuracy.

\section{Result of 3-D Simulation of Location Uncertainty under triangular,square and hexagonal distribution}

Fig. 19, Fig. 20, and Fig. 21 show the distribution of location uncertainty in our three types of WLAN infrastructure in 3D space. This time is in a $3 \times 3 \times 3 \mathrm{~m}$ 3-D grid with a total of 17,340 grid points. The dots of poor positioning accuracy in general correspond to corners, edges and walls where signals overlap and propagate weakly. Fig. 19 shows 698 colored points in a triangular distribution in a $3-\mathrm{D}$ space $(150 \mathrm{~m} \times 100 \mathrm{~m} \times 10 \mathrm{~m})$. The positioning accuracy is approximately $96 \%$. Fig. 20 shows 514 colored points in a square distribution in a 3-D space. The positioning accuracy is approximately 97\%. Fig. 21 shows 220 colored points in hexagonal distribution in a $3 \mathrm{D}$ space. The positioning accuracy is approximately $98.7 \%$.

\section{E. Result for Trajectory Estimation}

Fig. 22 shows the original and estimated walking trajectory on the 7th floor PQ building at The Hong Kong Polytechnic University under Kalman Filter and Traditional Location Fingerprinting approach. As can be seen in Fig. 22, due to signal fluctuation, the estimated path of traditional LF bulged inside the room PQ703 and PQ717 sharply. We make use of our previous work [9] to partially eliminate inaccurate estimation due to signal fluctuation. The result indicates that Wi-Fi positioning approach could be stably and precisely locate a WLAN-enabled device. 


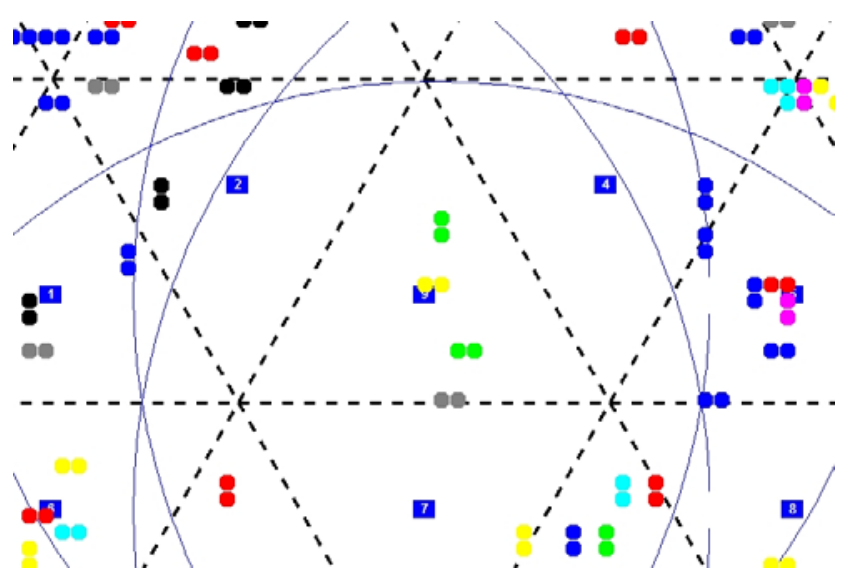

Fig. 16. The distribution of location uncertanity in a triangular WLAN Infrastructure

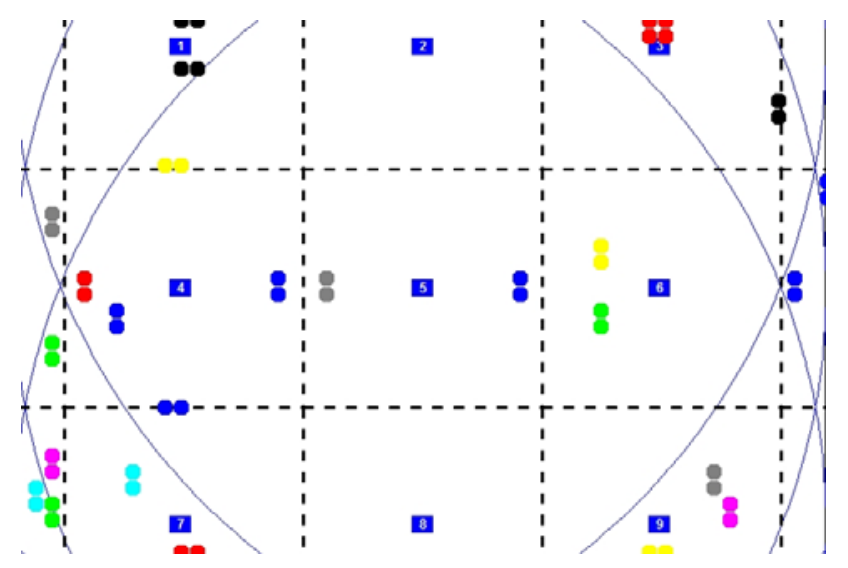

Fig. 17. The distribution of location uncertanity in a square WLAN Infrastructure

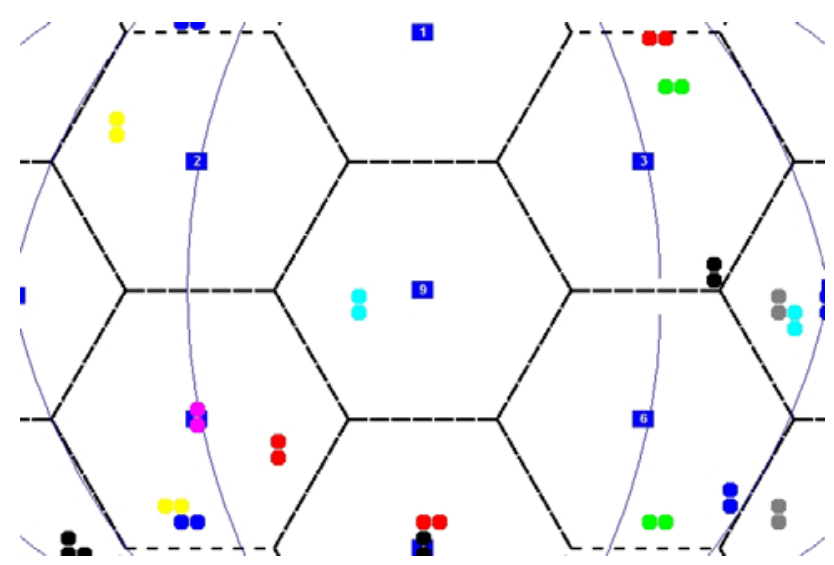

Fig. 18. The distribution of location uncertanity in a hexgonal WLAN Infrastructure

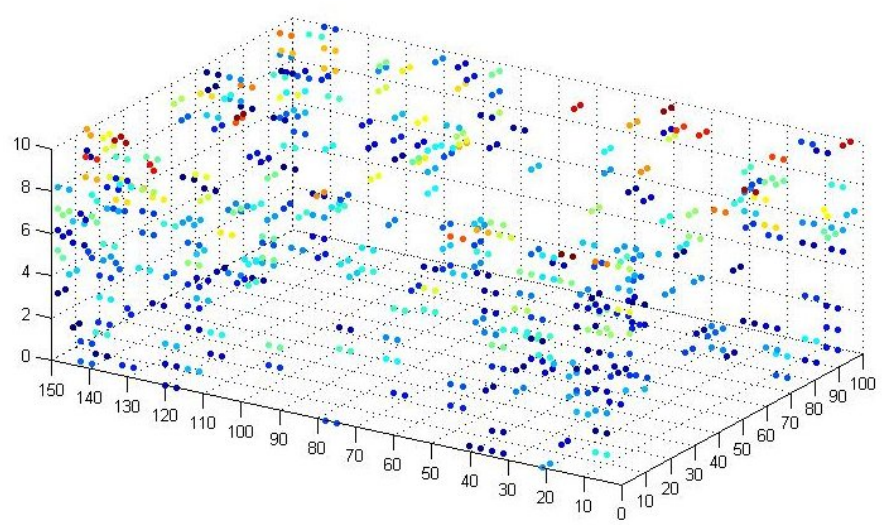

Fig. 19. 3-D Distribution of Location Uncertainty in triangular APs distribution

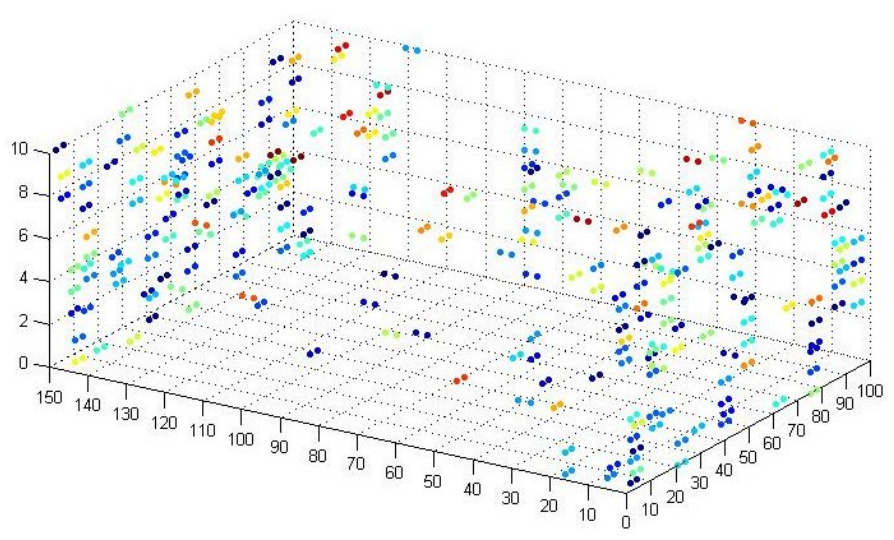

Fig. 20. 3-D Distribution of Location Uncertainty in square APs distribution

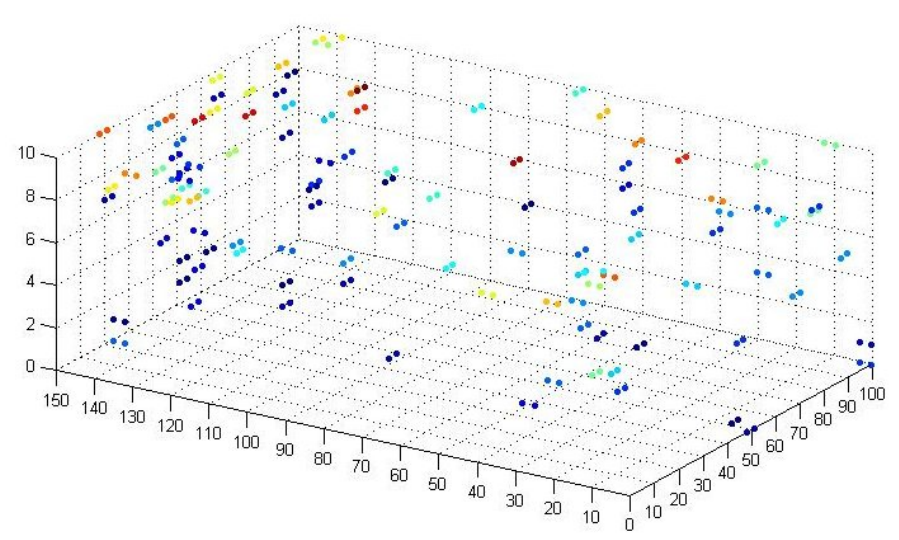

Fig. 21. 3-D Distribution of Location Uncertainty in hexagonal APs distribution 


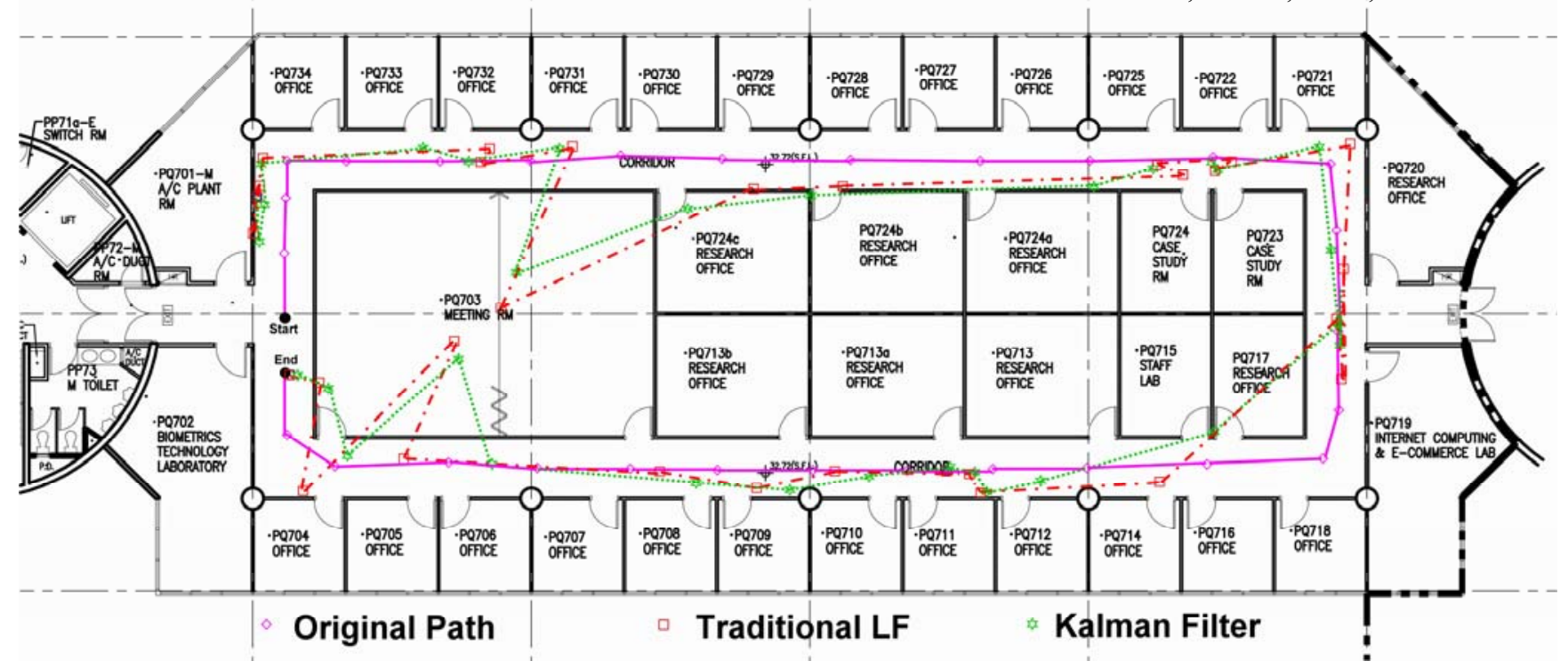

Fig. 22. Original and estimated walking trajectory on the 7th floor PQ building at The Hong Kong Polytechnic University under Kalman Filter and Traditional Location Fingerprinting approach

\section{F. $\quad$ RSS distribution on building floor}

Fig. 23 shows the RSS distribution on the 7th floor PQ building at The Hong Kong Polytechnic University, where red to blue represent as strong to weak signal strength. As be shown as Fig. 23, there is a red intensity cluster in the middle left of the floor plan (near the room PQ703), it means that the density of signal strength is strong at there, and the density of signal strength is weak at the right-bottom corner. Our sampling locations are even distributed, but the APs are not equally distributed. Signal distributes from strongly in the left to weakly in the right. This result indicates that the signal is not equally distributed even in an empirical real case.

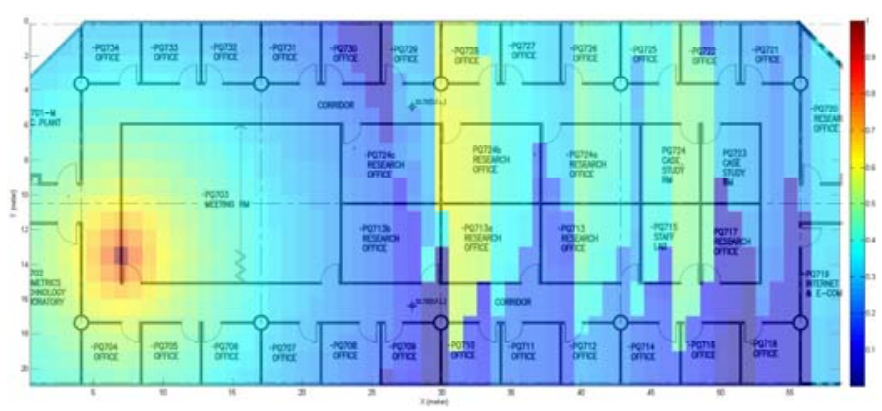

Fig. 23. Fuzzy WLAN Signal Distribution on the 7th floor PQ building at The Hong Kong Polytechnic University

\section{CONCLUSION}

A typical WLAN may cover a very large area, built up and topographically varied, and require the placement of many thousands of APs, for example, the area of The Hong Kong Polytechnic University is 9.53 hectares and is provided with more than 3,000 APs. Current approaches to the deployment of APS, however, are ad hoc or empirical and involve considerable applications of human and physical resources, especially where high positioning accuracy for a complete area of coverage is required. In this paper, we propose and simulate three geometric structured approaches to WLAN infrastructure deployment and in a series of simulations compare their accuracy with that of unstructured approaches. The contribution of the paper is that we show that the current unstructured approach to AP deployment is less cost effective than the structured approaches in our experiments. It is of interest to note, and may be useful to know in some circumstances, that where few APs are to be deployed, the triangular approach may outperform the hexagonal approach. Overall, however, the hexagonal approach is by far the most cost-effective approach and infrastructure designers and administrators may wish to take account of this in their infrastructure designs and deployments.

\section{XI.FUTURE WORK}

Although our proposed approach seems to give an improvement of positioning and shows structural deployment of AP is better than an empirical approach, the proposed approach suffers from physical limitations such as the depth of walls and floors and their composition (e.g. concrete) that would affect issues of AP placements and visualization of wireless signal. In real world scenarios, engineers need to go through every floor of every building in a construction site to detect whether a region is sufficient enough to be covered by wireless APs. After they collect wireless signal sample, they will decide where APs should be added to increase signal coverage or be taken away to reduce signal interference. As mentioned, strict implementation of our proposed approach would be too ideal because architecture of a building may not allow such AP deployment. Our proposed approach could be act as blueprint of deployment to save massive labor works for signal surveying. In the future, we will explore the hybrid deployment of structural and empirical approaches that allow the scalability and flexibility. We will make use of findings in wireless signal distribution and location uncertainty to look at energy-consumption problems of wireless infrastructure.

\section{REFERENCES}

[1] J.-H. Huang, L.-C. Wang, and C.-J. Chang, "Deployment strategies of access points for outdoor wireless local area networks," in Vehicular Technology Conference, 2005. VTC 2005-Spring. 2005 IEEE 61st, 2005, pp. 2949-2953. 
[2] C. Budianu, S. Ben-David, and L. Tong, "Estimation of the number of operating sensors in large-scale sensor networks with mobile access," in Signal Processing, IEEE Transactions, 2006, pp. 1703-1715.

[3] N. Swangmuang and P. Krishnamurthy, "Location Fingerprint Analyses Toward Efficient Indoor Positioning," in Sixth Annual IEEE International Conference, 2008, pp. 100-109.

[4] K. Kaemarungsi and P. Krishnamurthy, "Modeling of indoor positioning systems based on location fingerprinting," in INFOCOM 2004. Twenty-third AnnualJoint Conference of the IEEE Computer and Communications Societies, 2004, pp. 1012-1022.

[5] A. Taheri, A. Singh, and A. Emmanuel, "Location fingerprinting on infrastructure 802.11 wireless local area networks (WLANs) using locus," in Local Computer Networks, 2004, 29th Annual IEEE International Conference, 2004, pp. 676-683.

[6] J. Kwon, B. Dundar, and P. Varaiya, "Hybrid algorithm for indoor positioning using wireless LAN," 2004.

[7] B. Li, Y. Wang, H. K. Lee, A. Dempster, and C. Rizos, "Method for yielding a database of location fingerprints in WLAN," in Communications, IEE Proceedings, 2005, pp. 580586.

[8] C. L. Chan, G. Baciu, and S. C. Mak, "Wireless Tracking Analysis in Location Fingerprint," in IEEE Wireless and Mobile Computing, Networking and Communications, 2008, pp. 214220.

[9] C. L. Chan, G. Baciu, and S. C. Mak, "Using Wi-Fi Signal Strength to Localize in Wireless Sensor Networks," in IEEE International Conference on Communications and Mobile Computing, 2009, pp. 538-542.

[10] C. L. Chan, G. Baciu, and S. C. Mak, "Using the Newton TrustRegion Method to Localize in WLAN Environment," in 5th IEEE International Conference on Wireless and Mobile Computing, Networking and Communications., 2009, pp.363369.

[11] C. L. Chan, G. Baciu, and S. C. Mak, "Fuzzy Topographic Modeling in WLAN Tracking Analysis", accepted by International Conference on Fuzzy Computation.

[12] C. L. Chan, G. Baciu, and S. C. Mak, "Resource-effective and Accurate WLAN Infrastructure Design and Localization Using a Cell-structure Framework," in 5th International Conference on Wireless Communications, Network and Mobile Computing, 2009, pp. 9-15.

[13] I. Rubin and C. W. Choi, "Impact of the location area structure on the performance of signaling channels in wireless cellular networks," in Communications Magazine, IEEE, 1997, pp. 108115.

[14] R. H. Jan and Y. R. Lee, "An indoor geolocation system for wireless LANs," in Parallel Processing Workshops, 2003. Proceedings. 2003 International Conference, 2003, pp. 29-34.

[15] P. Prasithsangaree, P. Krishnamurthy, and P. K. Chrysanthis, "On indoor position location with wireless LANs," 2002, pp. 720-724.

[16] D. Dearman, A. Varshavsky, E. d. Lara, and K. N. Truong, "An Exploration of Location Error Estimation," in UbiComp 2007, 2007, pp. 181-198.

[17] H. Lemelson, M. B. Kjaergaard, R. Hansen, and T. King, "Error Estimation for Indoor 802.11 Location Fingerprinting," in Proceedings of the 4th International Symposium on Location and Context Awareness, 2009.

[18] J. Carle, J. Myoupo, and I. Stojmenovic, "Higher Dimensional Honeycomb Networks," in Journal of Interconnection Networks, 2001, pp. 391-420.

[19] K. Kaemarungsi and P. Krishnamurthy, "Properties of indoor received signal strength for WLAN location fingerprinting," in Mobile and Ubiquitous Systems: Networking and Services. MOBIQUITOUS 2004. The First Annual International Conference, 2004, pp. 14-23.

[20] K. Yoo and C. Kim, "A Channel Management Scheme for Reducing Interference in Ubiquitous Wireless LANs Environment," in Multimedia and Ubiquitous Engineering, 2008, 2008, pp. 276-281.

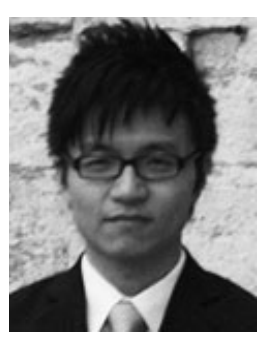

Eddie C. L. Chan received his BSc Degree in Computing and MSc Degree in E-commerce in the Department of Computing from The Hong Kong Polytechnic University in 2005 and 2007, respectively. Currently, he is a $\mathrm{PhD}$ student in the same university. His research interests include wireless communication, localization, fuzzy logic, 3D visualization of tracking system, agent technology and data mining. He is a member of the Graphics and Multimedia Applications (GAMA) Laboratory at The Hong Kong Polytechnic University.

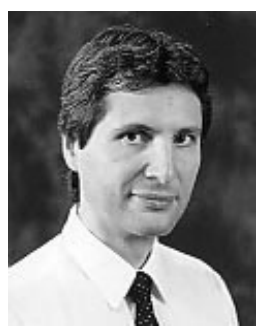

George Baciu holds a $\mathrm{PhD}$ and a MSc degree in Systems Engineering and a B.Math degree in Computer Science and Applied Mathematics from the University of Waterloo. He has been a member of the Computer Graphics Laboratory and the Pattern Analysis and Machine Intelligence Laboratory at the University of Waterloo and subsequently Director of the Graphics And Music Experimentation Laboratory at The Hong Kong University of Science and Technology in Hong Kong. Currently, Dr. Baciu is Professor and Associate Head in the Department of Computing and the founding director of the Graphics And Multimedia Applications (GAMA) Laboratory at The Hong Kong Polytechnic University. His research interests are primarily in mobile augmented reality systems, user interfaces, physically-based illumination, rendering, image processing, motion tracking and synthesis for both outdoor and indoor location aware systems.

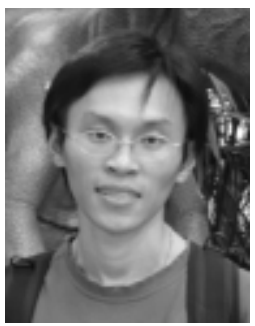

system.
S. C. Mak received his BSc Degree in Computing in the Department of Computing from The Hong Kong Polytechnic University in 2008. Currently, he is a member of the Graphics and Multimedia Applications (GAMA) Laboratory at The Hong Kong Polytechnic University. His research interests include wireless localization and tracking 